\title{
Atenção odontológica a pacientes especiais: atitudes e percepções de acadêmicos de odontologia
}

\author{
Marcela F. Sousa Santos*, Ignez A. dos Anjos Hora** \\ * Cirurgiã-dentista da Residência Multiprofissional em Saúde do \\ Adulto e Idoso da Universidade Federal de Sergipe, Aracaju, \\ Sergipe, Brasil \\ ** Mestre em Estomatologia e Especialista em Odontologia para \\ Pacientes Especiais da Universidade Federal de Sergipe, Aracaju, \\ Sergipe, Brasil
}

\section{RESUMO}

Estudo exploratório realizado em 2011 com o objetivo de conhecer sob a perspectiva dos acadêmicos do último ano de odontologia de Sergipe as abordagens realizadas durante a graduação, atitudes, percepções, e expectativas frente ao atendimento do paciente especial. Os resultados obtidos no questionário aplicado a 96 alunos mostram que as abordagens sobre odontologia para o paciente especial ocorreram para $76,7 \%$ em aulas expositivas e seminários nos períodos iniciais, e para $65,6 \%$ em congressos e projetos de extensão. Pacientes com alteração sistêmica foram atendidos por $48,9 \%$ dos alunos. Destacaram-se como percepções marcantes a vontade de ajudar e, de estudar mais o paciente especial, relatadas por $51,6 \%$ e $49,4 \%$ respectivamente. Para 93,7\% há necessidade da implantação da disciplina odontologia para pacientes especiais nas faculdades, e 56,7\% referem insegurança frente ao atendimento futuro destes. Conclui-se que há necessidade de intensificação e diversificação de abordagens dentro das instituições estudadas, promovendo maior conhecimento e capacitação acadêmica, e favorecendo a atenção odontológica e maior inclusão dos pacientes especiais.

\section{DESCRITORES}

Pacientes Especiais. Atitudes. Estudantes de Odontologia.

A odontologia para pacientes especiais, atualmente pautada em bases científicas, busca uma abordagem ampla e integrada. Assim, reconhece-se a importância de práticas clínicas motivadoras du- rante a graduação, que preparem o acadêmico para o futuro profissional visando um atendimento satisfatório destes pacientes. ${ }^{1}$

Denomina-se especial o paciente que possui desvios nos padrões de normalidade, identificáveis ou não, que tornem necessário o atendimento diferenciado durante um período, ou por toda a sua vida. ${ }^{2}$

Em razão de suas limitações físicas, mentais e sociais, indivíduos com necessidades especiais tendem a apresentar maior comprometimento da saúde bucal. Assim, necessitam de uma atenção odontológica especial, com cuidados específicos de acordo com cada caso. ${ }^{3,4}$

De acordo com Organização Mundial de Saúde (OMS), a prevalência mundial de pessoas com deficiência é de 1:10 indivíduos. ${ }^{4}$ No Brasil, $15 \%$ da população possui necessidades especiais, com o maior porcentual concentrado na Região Nordeste $(16,8 \%) .5$

Em contraste com a alta demanda, é restrito no país, o número de pacientes especiais que têm acesso ao atendimento. O Instituto Brasileiro de Geografia e Estatística (IBGE) estima que de $10 \%$ das pessoas com necessidades especiais, apenas $3 \%$ recebem atendimento odontológico, o correspondente a 480 mil pacientes. ${ }^{4,6,7}$

As Diretrizes Curriculares Nacionais para os cursos de Odontologia no Brasil, vigentes desde 2002, determinam que a formação do cirurgião-dentista deve capacitá-lo a atuar em todos os níveis de atenção à saúde e, para isso, deve haver uma formação generalista. É importante que esta inclua a atenção odontológica ao paciente especial. ${ }^{8}$

É limitado o número de cursos de odontologia 
no Brasil que proporcionam aos graduandos espaço físico e treinamento específico para tratar pacientes especiais. Assim, os alunos muitas vezes não têm a oportunidade de ter maior contato com estes pacientes. ${ }^{4}$ Os cursos de odontologia de Sergipe se enquadram nesta realidade.

O estudo objetivou analisar as perspectivas acadêmicas sobre a ocorrência de abordagens acerca do atendimento odontológico ao paciente especial, e conhecer atitudes e percepções do alunado frente ao atendimento do mesmo.

\section{MATERIAL E MÉTODOS}

Estudo quantitativo, prospectivo e exploratório, realizado por meio de questionário fechado aplicado aos alunos do último ano dos cursos de odontologia da Universidade Federal de Sergipe (UFS), instituição pública, e da Universidade Tiradentes (UNIT), instituição particular, no período de maio a agosto de 2011. Ressalta-se que as instituições selecionadas são as únicas que possuem o curso de odontologia em Sergipe, mas não possuem a disciplina de Odontologia para pacientes especiais.

Após aprovação do projeto pelo Comitê de Ética em Pesquisa (sob o número CAAE - 0042.0.107.11911), e pelas coordenações de curso das instituições selecionadas, iniciou-se a identificação e seleção dos participantes.

Conforme critério de inclusão, os acadêmicos selecionados estavam no último ano do curso de odontologia das faculdades selecionadas. Pertenciam aos critérios de exclusão, aqueles acadêmicos que não estavam no último ano dos cursos, e também, aqueles que não concordaram em participar do estudo.

A amostra total da pesquisa foi de 96 acadêmicos, que após a explicação do objetivo e metodologia se encontraram em conformidade com os critérios de inclusão, assinaram o Termo de Consentimento Livre e Esclarecido e receberam o questionário fechado.

Após a coleta dos questionários, foi feita a organização dos dados com o auxílio do programa Microsoft Excel 2007, e quando necessário, foi aplicado o teste estatístico qui quadrado aplicado no programa Spss Statistica.

\section{RESULTADOS}

Da amostra composta por 96 acadêmicos $(91,4 \%)$ do total de matriculados no último ano de odontologia, $43(44,8 \%)$ pertenciam à Universidade Federal de Sergipe (UFS - Instituição A) e 53 (55,2\%) estudantes pertenciam à Universidade Tiradentes (UNIT - Instituição B).

A maioria dos participantes era do gênero feminino totalizando $61(63,5 \%)$ estudantes. No quesito idade, a maior parte pertencia à faixa etária de 21 a 23 anos correspondendo a $60(62,5 \%)$ alunos.

Os acadêmicos contemplados de alguma forma com a abordagem do tema odontologia para pacientes especiais totalizaram 60 alunos $(60 \%)$. A Tabela 1 apresenta o tipo de abordagem realizada nas duas instituições.

Os principais tipos de pacientes especiais mais atendidos pelos acadêmicos estão representados no Gráfico 1. A maioria dos participantes atendeu pacientes com alteração sistêmica $(48,9 \%)$ e gestantes $(35,4 \%)$.

Tabela 1 - Formas de abordagens realizadas.

\begin{tabular}{|l|c|c|}
\hline $\begin{array}{c}\text { Formas de } \\
\text { abordagens }\end{array}$ & No de Alunos & $\%$ \\
\hline Aula expositiva & 31 & $51,7 \%$ \\
\hline Seminário & 15 & $25,0 \%$ \\
\hline Pesquisa & 5 & $8,3 \%$ \\
\hline Estudo dirigido & 3 & $5,0 \%$ \\
\hline Outro tipo & 6 & $10,0 \%$ \\
\hline *Total & 60 & $100 \%$ \\
\hline
\end{tabular}

${ }^{* \star}$ Do total de 96 alunos participantes somente 60 receberam a abordagem.

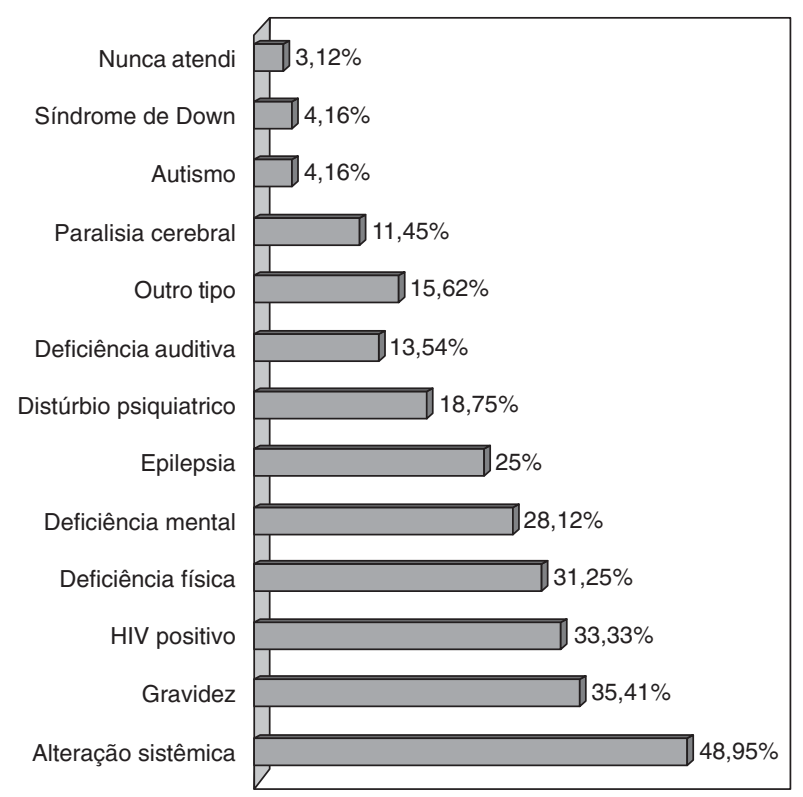

Gráfico 1 - Percentuais de acadêmicos quanto aos principais tipos de pacientes especiais atendidos. 
O Gráfico 2, reflete as percepções e sentimentos relatados pelos acadêmicos ao atender nas disciplinas ambulatoriais o paciente especial; têm destaque a vontade de ajudar $(51,6 \%)$ e de estudar mais o paciente especial $(49,4 \%)$.

Outro dado importante é que 90 alunos (94\%), afirmaram que a implantação da disciplina de Odontologia para pacientes especiais seria necessária para melhorar o seu aprendizado, enquanto apenas 6 participantes $(6 \%)$ relataram não sentir essa necessidade.

Sobre a acessibilidade do paciente especial ao atendimento odontológico, 90 participantes (94\%) referem que haja dificuldade no acesso. Para eles, isso se deve principalmente ao desconhecimento da população sobre os serviços e à escassez de profissionais disponíveis para a realização do atendi-

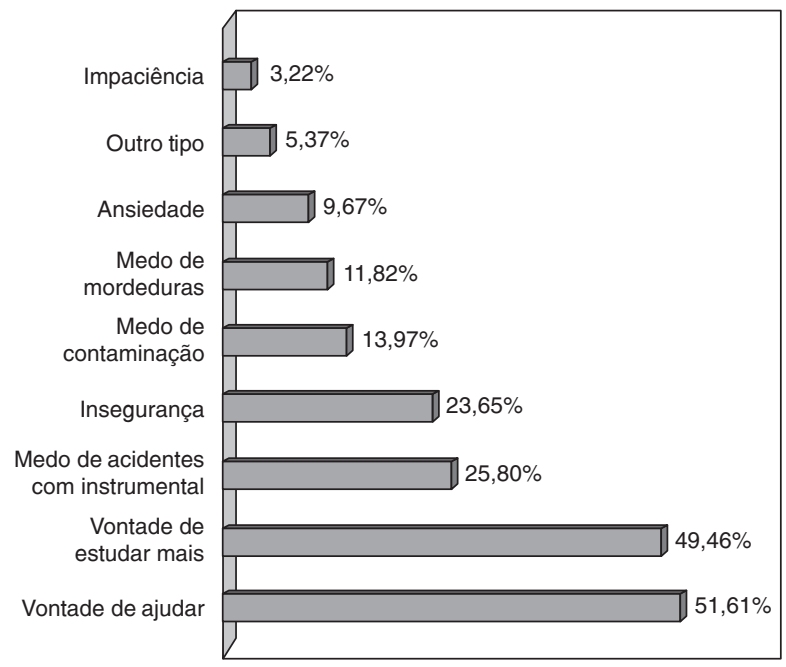

Gráfico 2 - Percepções e sentimentos do acadêmico frente ao paciente especial. mento (Gráfico 3).

Apesar de 60 estudantes $(62,5 \%)$, receberem a abordagem do tema Odontologia para pacientes especiais, na graduação, vê-se na Tabela 2, que apenas as abordagens, não apresentaram significância estatística $(p>0,05)$ para gerar segurança no atendimento do paciente especial. Mas, pode-se afirmar que houve influência positiva dessas abordagens para atendimento odontológico relatada pelos alunos contemplados $(20 \%)$.

\section{DISCUSSÃo}

Ressalta-se que na literatura consultada, não foram encontrados todos os dados que pudessem ser comparados com os resultados deste trabalho, uma vez que ainda é reduzido o número de pesquisas que abordem exatamente os mesmos aspectos estudados.

Tabela 2 - Abordagem do tema relacionada à segurança no atendimento futuro.

\begin{tabular}{|c|c|c|c|c|c|}
\hline \multirow{2}{*}{ Segurança } & \multicolumn{5}{|c|}{ Abordagem do tema } \\
\cline { 2 - 7 } & Sim & $\%$ & Não & $\%$ & Total \\
\hline Sim & 12 & $20,0 \%$ & 5 & $13,9 \%$ & 17 \\
\hline Não & 34 & $56,7 \%$ & 25 & $69,4 \%$ & 59 \\
\hline Não sei & 14 & $23,3 \%$ & 6 & $16,7 \%$ & 20 \\
\hline Total & 60 & $100 \%$ & 36 & $100 \%$ & 96 \\
\hline
\end{tabular}

${ }^{*} \alpha=5 \% ;{ }^{* *} p=0,46$. De acordo com o resultado do teste $(p>0,05)$ não há associação entre as abordagens realizadas e segurança no atendimento futuro, visto que entre os treinados a maioria $(56,7 \%)$ afirmou não ter segurança no atendimento destes pacientes. Também concluímos com os resultados da pesquisa que a abordagem teve alguma utilidade, comprovada por uma maior $(20,0 \%)$ segurança de atendimento entre os que receberam a abordagem nos cursos.

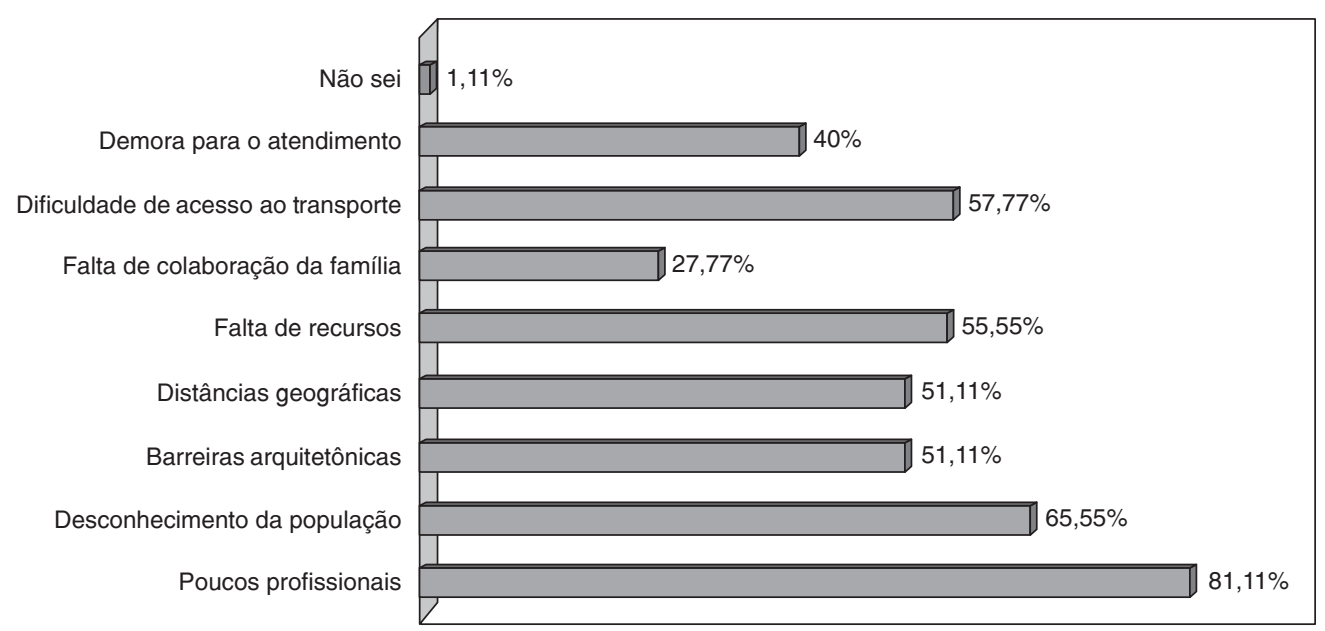

Gráfico 3 - O que dificulta o acesso ao atendimento odontológico do paciente especial? 
Atenção odontológica a pacientes especiais: atitudes e percepções de acadêmicos de odontologia $\bullet$ Santos MFS, Hora IAA

No Brasil, ainda são poucas as faculdades de odontologia que proporcionam aos graduandos o preparo adequado e específico para o atendimento de pacientes com necessidades especiais. ${ }^{1,2}$ A maioria das escolas dedica pouco tempo para este atendimento. ${ }^{9}$ Nas faculdades selecionadas, não há nenhum programa de ensino específico para o atendimento do paciente especial.

Os participantes, que foram contemplados pelas abordagens, receberam-nas no início da prática clínica. Estas se apresentaram em eventuais aulas expositivas ou seminários. Em acordo com os autores, que relatam que a maioria dos cursos dedica poucas horas para a discussão sobre deficiências, tempo insuficiente para que o estudante desenvolva as habilidades e segurança necessária para o cuidado de tais pacientes. ${ }^{1,8}$

Os autores consultados salientaram a necessidade que o aluno tem de conhecer sobre o paciente especial para que se sensibilize e adquira conhecimento específico para o tratamento odontológico. ${ }^{7,10,11,12}$ Isso é relatado pela maioria dos acadêmicos, que referem necessidade de estudar mais sobre este paciente.

Grande parte dos alunos tem ciência de que a demanda do atendimento odontológico para o paciente é grande, entretanto, acredita que os serviços existentes são insuficientes para suprir a mesma, ou até mesmo desconhece se estes serviços têm a capacidade de suprir a demanda.

Os resultados mostram a importância relatada pelos acadêmicos, de possuírem em seu curso, a disciplina de odontologia para pacientes especiais. Proporcionar ao acadêmico a possibilidade de conhecer sobre pacientes especiais, é aspecto relevante no tocante ao conhecimento do futuro profissional. ${ }^{8}$ Esta vontade de estudar mais sobre o tema também aparece em alto porcentual.

É importante que os acadêmicos conheçam as dificuldades que impedem o paciente de ter o acesso odontológico, seja ele de qualquer natureza. ${ }^{4}$ Muitos acadêmicos consultados, reconhecem que há dificuldades neste acesso e os tipos de dificuldade existentes.

O cirurgião-dentista pode ter importante contribuição para a qualidade de vida do paciente especial. Porém, o que se observa frequentemente, é a insegurança de muitos em promover o atendimento, principalmente devido à falta de experiência na academia. ${ }^{6}$ Isso é notado em muitos participantes. As abordagens recebidas foram importantes, mas, insu- ficientes para gerar segurança significante para um futuro atendimento profissional.

Outro dado importante é que a maioria do alunado afirma que a implantação da disciplina de Odontologia para pacientes especiais seria necessária para melhorar o seu aprendizado.

Sobre a acessibilidade do paciente especial ao atendimento odontológico, 90 participantes (94\%) acreditam que este tenha dificuldade no acesso ao atendimento odontológico. Para eles, isso se deve especialmente ao desconhecimento da população sobre os serviços e à escassez de profissionais disponíveis para a realização do atendimento.

Vê-se, que apenas as abordagens realizadas, não revelaram significância estatística $(p>0,05)$ para gerar segurança no atendimento futuro do paciente especial. Entretanto, pode-se afirmar que houve influência positiva destas relatada pelos participantes que foram contemplados $(20 \%)$.

As abordagens ocorreram quando os alunos estavam no início da academia, principalmente em aulas expositivas e seminários. O que coincide com achados de autores que relatam que a maioria dos cursos de graduação dedica poucas horas para discussões sobre o assunto. ${ }^{1,8}$

A literatura salientou a necessidade que o aluno tem de conhecer sobre o paciente especial para que haja sensibilização e conhecimento específico para o tratamento odontológico. ${ }^{7,10,11,12}$ Tal necessidade é mencionada pela maioria dos alunos consultados.

Muitos participantes referem que a demanda do atendimento odontológico para o paciente é grande, mas, acredita que os serviços existentes são insuficientes para suprir a mesma, ou até mesmo desconhece se estes serviços têm a capacidade de supri-la.

É importante que os acadêmicos conheçam as dificuldades que impedem o paciente de ter o acesso odontológico, seja ele de qualquer natureza. ${ }^{4}$ Muitos dos acadêmicos consultados, reconhecem que há dificuldades neste acesso e referem os tipos de dificuldade existentes.

Estudos mostram que os profissionais durante a graduação tiveram treinamento específico para o atendimento odontológico de pacientes especiais não tinham obstáculos para o mesmo. ${ }^{7}$ Nesta pesquisa, apesar de a maioria acadêmica relatar ter atendido pacientes especiais, nem todos tiveram contato com abordagens específicas.

Nos cursos de odontologia no Brasil, há uma lacuna em relação à formação profissional para atender a pessoas com deficiências físicas e mentais, as- 
Atenção odontológica a pacientes especiais: atitudes e percepções de acadêmicos de odontologia $\bullet$ Santos MFS, Hora IAA

sim, os cirurgiões-dentistas não se sentem seguros e capacitados para o atendimento. ${ }^{8}$ Os resultados destacam a necessidade acadêmica, de possuir a disciplina de odontologia para pacientes especiais na graduação, mostrando uma maior necessidade de contato destes alunos com o tema.

O aluno pode ter importante contribuição para o paciente especial. Porém, o que se observa, é a insegurança de muitos em promover o atendimento odontológico deste paciente, principalmente devido à falta de experiência na academia. ${ }^{5}$ Isso é notado em muitos participantes que tiveram ou não contato com o tema Odontologia para pacientes especiais nesta pesquisa. As abordagens foram relevantes, porém, ainda insuficientes para gerar segurança para o futuro atendimento profissional de boa parte do alunado.

\section{Conclusões}

De acordo com os resultados obtidos, pode-se concluir que há necessidade de intensificação e diversificação de abordagens sobre atendimento odontológico ao paciente especial dentro das faculdades de Odontologia de Sergipe, bem como, de proporcionar maior contato com pacientes especiais e inclusão destes, principalmente aqueles de maior complexidade para ampliar a experiência dos alunos, uma vez que o anseio de conhecimento e a insegurança dos acadêmicos frente ao paciente especial, bem como a necessidade relatada por uma disciplina específica são percepções marcantes no estudo.

\section{AGRADECIMENTOS}

Agradecemos às coordenadorias dos cursos de Odontologia da Universidade Federal de Sergipe e da Universidade Tiradentes bem como, a todo o público acadêmico que fez parte desta pesquisa. Também agradecemos ao professor Samuel Oliveira Ribeiro pelo apoio dado na organização estatística do estudo, e a todos aqueles que, direta ou indiretamente contribuíram para a concretização desta pesquisa.

\section{ABSTRACT}

Dental care for special-needs patients: attitudes and perceptions of dentistry students

This was an exploratory study conducted in 2011 aimed at gaining more knowledge into the approaches used in the dentistry course, and into attitudes, perceptions and expectations toward the care extended to special-needs dental patients, from the perspective of last-year undergraduates from the Federal University of Sergipe Dentistry Course. The results of a questionnaire applied to 96 students show that, to $76.7 \%$ of these students, the approaches to dental issues regarding special-needs patients were conveyed in lectures and seminars early in the learning process, and, to $65.6 \%$, at conferences and in outreach projects. Patients with systemic illnesses received dental care by $48.9 \%$ of the students. The importance of wanting to help special-needs patients and of studying more about the correct approaches for these patients stood out as strong perceived by $51.6 \%$ and $49.4 \%$ of the students, respectively. According to $93.7 \%$, there is a need to establish a college course addressing "dental care for special-needs patients," and, according to $56.7 \%$ of the students, they were still not prepared to treat this kind of patient in the future. It was concluded that what is needed is to intensify and diversify the approaches in the institutions studied, promoting greater knowledge and academic training with the aim of providing improved dental care and a greater inclusion of special-needs patients.

\section{DESCRIPTORS}

Special-needs Patients. Attitudes. Students, Dental. -

\section{REFERÊNCIAS}

1. Fassina AP. Presença da disciplina e/ou conteúdo de pacientes portadores de necessidades especiais nas Faculdades de Odontologia no Brasil em 2005. [dissertação de mestrado em odontologia]. São Paulo: Odontologia e Sociedade; 2007.

2. Paulo JR, Acessibilidade e participação de pacientes com necessidades especiais nos serviços públicos odontológicos do município de João Pessoa. [trabalho de conclusão de curso]. João Pessoa: Universidade Federal da Paraíba; 2010.

3. Dall'Magro AK, Dall'Magro E, Kuhn GF. The clinic profile of patients with special needs treated with general anesthesic in the São Vicente de Paulo Hospital of Passo Fundo between 2005 and 2010. RFO 2010; 15(3)253-56.

4. Fukuoka CY, Crosato EM, Filho IE, Biazevic MG, Zaitter WM. Accessibility to dental care for patients with special needs. RSBO 2011; 8(3):277-81.

5. Cancino CM, Oliveira FA, Engers ME, Weber JB, Oliveira MG. Dentistry for patients with special needs: feelings, perceptions and reactions of students and handicapped patients. [tese de doutorado em odontologia]. Porto Alegre: Pontifícia Universidade Católica do Rio Grande do Sul; 2006.

6. Castro AM, Marchesoti MG, Oliveira FS, Novaes MS. Avaliação do tratamento odontológico de pacientes com necessidades 
especiais sob anestesia geral. Rev. Odontol. UNESP 2010; $39(3): 137-42$.

7. Oliveira ET, Júnior JF, Soares FN, Maia ER. Social dentistry in the context of Health Promotion. RBPS 2008; 21(1):75-9.

8. Ditterich RG, Portero PP, Schmidt LM. A preocupação social nos currículos de odontologia. ABENO 2007;7(1):58-62.

9. Gomes MJ, Caxias FP, Margon CD, Rosa RG, Carvalho RB. A percepção dos docentes do Curso de Odontologia da UFES em relação à necessidade de inclusão da disciplina denominada "Atendimento Odontológico a Pacientes Portadores de Necessidades Especiais".Revista Brasileira de Pesquisa em Saúde 2009; 11(1):33-9. 2009.

10. Schwenk DM, Stoeckel DC, Rieken SE. Survey of Special Pa- tient Care Programs at U.S. and Canadian Dental Schools. Journal of Dental Education 2007; 71(9): 1153-59.

11. Cordioli OF, Batista NA. A graduação em odontologia na visão de egressos: propostas de mudanças. Abeno 2007; 7(1):8895.

12. Fernandes IM. Acolhimento a portadores de necessidades especiais nos Centros de Especialidades Odontológicas do município de João Pessoa. [trabalho de conclusão de curso]. João Pessoa: Universidade Federal da Paraíba; 2009.

Recebido em 08/10/2012

Aceito em 10/12/2012 